Article

\title{
Pharmacokinetic/Pharmacodynamic (PK/PD) Simulation for Dosage Optimization of Colistin Against Carbapenem-Resistant Klebsiella pneumoniae and Carbapenem-Resistant Escherichia coli
}

\author{
Kamonchanok Jitaree ${ }^{1}$, Korbtham Sathirakul ${ }^{1}{ }^{(\mathbb{0}}$, Jantana Houngsaitong ${ }^{1}$, Orarik Asuphon ${ }^{2}$, \\ Weerayuth Saelim ${ }^{3}$, Visanu Thamlikitkul ${ }^{4, *}$ and Preecha Montakantikul ${ }^{5, *}$ \\ 1 Department of Pharmacy, Faculty of Pharmacy, Mahidol University, Bangkok 10400, Thailand \\ 2 Department of Pharmacy Practice, Faculty of Pharmaceutical Sciences, Naresuan University, \\ Phitsanulok 65000, Thailand \\ 3 Department of Pharmacy, Faculty of pharmacy, Silpakorn University, Nakhon Pathom 73000, Thailand \\ 4 Division of Infectious Disease and Tropical Medicine, Faculty of Medicine, Siriraj Hospital, \\ Mahidol University, Bangkok 10700, Thailand \\ 5 Department of Pharmacy, Faculty of Pharmacy, Mahidol University, Bangkok 10400, Thailand \\ * Correspondence: visanu.tha@mahidol.ac.th (V.T.); preecha.mon@mahidol.ac.th (P.M.); \\ Tel.: +66-2-419-7767-69 (V.T.); +66-2644-8694 (P.M.)
}

Received: 17 July 2019; Accepted: 16 August 2019; Published: 22 August 2019

\begin{abstract}
The purpose was to explore the optimal dosage regimen of colistin using Monte Carlo simulations, for the treatment of carbapenem-resistant Klebsiella pneumoniae and carbapenem-resistant Escherichia coli based on PK/PD targets in critically ill patients. A total of 116 carbapenem-resistant K. pneumoniae and E. coli were obtained from various clinical specimens at Siriraj Hospital in Bangkok, Thailand. Minimum inhibitory concentrations (MICs) of colistin were determined by broth microdilution method. Monte Carlo simulation was used to calculate the cumulative fraction of response (CFR) for European Medicine Agency (EMA), US-Food and Drug Administration (FDA), Nation et al., Siriraj Hospital and our study regimens. The targeted CFR was $90 \%$. For colistin-susceptible K. pneumoniae, all of the dosage regimens achieved $\geq 90 \%$ CFR in patients with creatinine clearance $<80 \mathrm{~mL} / \mathrm{min}$ except the FDA-approved regimens for patients with creatinine clearance $51-79$ and $11-29 \mathrm{~mL} / \mathrm{min}$, respectively. While, patients with creatinine clearance $\geq 80 \mathrm{~mL} / \mathrm{min}$, CFR $\geq 90 \%$ was observed in Siriraj Hospital and our study regimen. For colistin-susceptible E. coli, all of the dosage regimens achieved $\geq 90 \%$ CFR regardless of renal function. In contrast, the currently approved regimens achieved CFR target in only $10-50 \%$ for colistin-resistant isolates subgroup. These results suggest that currently approved regimens still recommended for colistin-susceptible CRE. For colistin-resistant CRE, alternative approaches such as high dose or combination therapy should be considered.
\end{abstract}

Keywords: colistin; Monte Carlo simulation; pharmacokinetics; pharmacodynamics; carbapenem-resistant Klebsiella pneumoniae; carbapenem-resistant Escherichia coli

\section{Introduction}

Worldwide emergence of carbapenem-resistant gram-negative bacteria, and particularly carbapenem-resistant Enterobacteriaceae (CRE) have become a major concern [1]. Specifically, carbapenem-resistant Klebsiella pneumoniae and carbapenem-resistant Escherichia coli which most commonly cause hospital acquired infections like intra-abdominal infections, urinary tract infections, 
nosocomial pneumonia, and bloodstream infections with high mortality rate of $44 \%$ to $70 \%$ [2,3]. In Thailand, the prevalences of carbapenem-resistant $K$. pneumoniae as well as carbapenem-resistant E. coli were approximately $2-10 \%$ in 2017 and these had rapidly increased from $0.3-0.8 \%$ in 2010 [4-6]. Concomitantly, there has been a shortage of newer antibiotics to fight multidrug-resistant (MDR) bacteria development. This has led to re-emergence of previously used old antibiotics like colistin.

Colistin has been developed as a mainstay of treatment for patients infected by carbapenem-resistant Enterobacteriaceae [7,8]. Although, optimal dosage regimen for colistin has been unclear because accurate pharmacodynamic and pharmacokinetic information are lacking. However, recent studies have improved our understanding about the pharmacokinetic/pharmacodynamic (PK/PD) which has guided us to selecting suitable colistin dosage regimen to treat carbapenem-resistant Enterobacteriaceae infections [7-9]. Suitable dosing strategies has lead to maximize the clinical efficacy, and to reduce the developing bacterial resistance rate, morbidity and mortality.

This study aimed to explore the optimal dosage regimen of colistin using Monte Carlo simulations, for the treatment of carbapenem-resistant K. pneumoniae and carbapenem-resistant E. coli based on $\mathrm{PK} / \mathrm{PD}$ targets in critically ill patients.

\section{Materials and Methods}

\subsection{Microbiology}

Carbapenem-resistant K. pneumoniae and carbapenem-resistant E. coli were obtained from various clinical specimens (sputum, urine, skin and soft tissue, blood, and pleural fluid) which were submitted for culture to the microbiological laboratory at the Faculty of Medicine at Siriraj Hospital in Bangkok, Thailand. The isolates were collected between June 2011 and September 2016. The Carbapenem-resistant Enterobacteriaceae (CRE) phenotype was identified for isolates expressing non-susceptible (intermediate or resistant) to any carbapenem antibiotics or documented to produce carbapenemase. Minimum inhibitory concentrations (MICs) of colistin were determined by the broth microdilution method in cation-adjusted Mueller-Hinton II broth (CA-MHBII) according to Clinical and Laboratory Standards Institute (CLSI) guidelines [10]. Colistin was tested over a range of dilutions from $0.25 \mathrm{mcg} / \mathrm{mL}$ to $128 \mathrm{mcg} / \mathrm{mL}$. Escherichia coli ATCC 25922 was used as a control. Isolate preparation was performed following the Clinical and Laboratory Standards Institute protocol (CLSI) [10]. The MIC value was defined as the lowest drug concentration for which the well was optically clear. The breakpoints of the European Committee on Antimicrobial Susceptibility Testing (EUCAST) (MIC $\leq 2 \mathrm{mcg} / \mathrm{mL}$ ) were applied to interpret those for colistin [11].

\subsection{Pharmacokinetic Model}

The population pharmacokinetic model for colistimethate sodium (CMS) and colistin were two-compartment and one-compar tment models, respectively. Pharmacokinetic data from Nation et al. [12] was retrieved from previously published studies concerning critically ill patients. Table 1 a set of parameters was randomly generated for each mean and standard deviation of the parameters. Equations (1)-(3) below represent the differential equations for the disposition of CMS and colistin.

$$
\begin{gathered}
\frac{\mathrm{dCMSc}}{\mathrm{dt}}=\mathrm{R} 1-\operatorname{CLD} 1 X\left(\frac{\mathrm{CMSc}}{\mathrm{V} 1}-\frac{\mathrm{CMSp}}{\mathrm{V} 2}\right)-\left(\text { CLTcms } X \frac{\mathrm{CMSc}}{\mathrm{V} 1}\right) \\
\frac{\mathrm{dCMSp}}{\mathrm{dt}}=\mathrm{CLD} 1 \times\left(\frac{\mathrm{CMSc}}{\mathrm{V} 1}-\frac{\mathrm{CMSp}}{\mathrm{V} 2}\right) \\
\frac{\mathrm{dColistin}}{\mathrm{dt}}=\left(\text { CLNRcms } X \frac{\mathrm{CMSc}}{\mathrm{V} 1}\right)-\left(\text { CLTc } X \frac{\text { Colistin }}{\mathrm{V} 3}\right)
\end{gathered}
$$

CMSc $=$ mass of CMS in the central compartment, CMSp = mass of CMS in the peripheral compartment, Colistin = mass of colistin in the single compartment, $\mathrm{V} 1=$ central volumes for CMS, V2 = peripheral volumes for CMS, V3 = volume of distribution for colistin, $\mathrm{R}(1)=$ infusion rate of CMS, 
CLD1 = distributional clearance between the central and peripheral compartments for CMS. CLTcms = total intrinsic clearance for CMS, CLTc = total intrinsic clearance for colistin and CLNRcms = nonrenal clearance for CMS.

Table 1. Population pharmacokinetic parameters.

\begin{tabular}{|c|c|c|c|c|c|}
\hline Category & Parameter & Units & Estimate & $\% S E$ & $\%$ IIV \\
\hline \multirow{5}{*}{ CMS } & $\mathrm{V} 1$ & $\mathrm{~L}$ & 12.9 & - & 40.4 \\
\hline & V2 & $\mathrm{L}$ & 16.1 & - & 70.9 \\
\hline & CLD1 & $\mathrm{L}$ & 9.57 & 10.5 & 80.1 \\
\hline & CLR & $\mathrm{L} / \mathrm{h} / \mathrm{CrCL}$ & 0.0340 & 6.85 & 75.2 \\
\hline & CLNR $_{\mathrm{CMS}}$ & $\mathrm{L} / \mathrm{h}$ & 2.52 & 3.71 & 39.8 \\
\hline \multirow{4}{*}{ Colistin } & V3 & $\mathrm{L}$ & 57.2 & 5.13 & 43.5 \\
\hline & $\mathrm{CLT}_{\mathrm{C}}$ & $\mathrm{L} / \mathrm{h}$ & 3.59 & - & 37.9 \\
\hline & $\mathrm{CLR}_{\mathrm{C}}$ & $\mathrm{L} / \mathrm{h} / \mathrm{CrCL}$ & 0.00834 & 27.7 & - \\
\hline & $\mathrm{CLNR}_{\mathrm{C}}$ & $\mathrm{L} / \mathrm{h}$ & 3.11 & 4.38 & - \\
\hline
\end{tabular}

V1 = Central volume for CMS, V2 = Peripheral volume for CMS, CLD1 = Distributional clearance between the central and peripheral compartments for CMS, CLR $=$ Renal clearance of $C M S, C L N R_{C M S}=$ Non-renal clearance of CMS, V3 = Volume of distribution of formed colistin, $C L T C=$ Total clearance of colistin, $C_{C} R_{C}=$ Renal clearance of colistin, $\mathrm{CLNR}_{\mathrm{C}}=$ Non-renally of colistin clearance, $\% \mathrm{SE}=$ The standard error or the precision of the estimates, $\% \mathrm{IIV}=$ The inter-individual variability in the population, $\mathrm{CrCL}=$ creatinine clearance.

\subsection{Pharmacodynamic Model}

The pharmacodynamic (PD) surrogate indices for colistin are characterized by area under the unbound colistin plasma concentration-time curve to the minimum inhibitory concentration ( $f$ AUC/MIC) [13,14]. PK/PD targets were defined as $f$ AUC/MIC $\geq 25$ [13]. The trapezoidal rule was used to calculate AUC, then $f$ AUC was divided by the MIC to obtain the value for the desired PK/PD index. A colistin unbound fraction of $50 \%$ was used [15]. The regimen administrated using IV infusions (infused over $30 \mathrm{~min}$ ) were simulated over a 24 -h period at different levels of renal function. The ranges of creatinine clearance $(\mathrm{CrCl})$ used were $\geq 80,51$ to 79,30 to 50,11 to 29 and $\leq 10 \mathrm{~mL} / \mathrm{min}$. The other dose was shown in Supplementary Materials Table S1.

The regimens were chosen by using European Medicine Agency (EMA), US-Food and Drug Administration (FDA), Nation et al., Siriraj Hospital and our study regimens Tables 2 and 3 [12,16-18].

Table 2. Colistin dosage regimens for Simulations.

\begin{tabular}{|c|c|c|}
\hline Creatinine Clearance & \multicolumn{2}{|c|}{ Daily Dose (CBA) } \\
\hline \multirow{3}{*}{$\geq 80 \mathrm{~mL} / \mathrm{min}$} & 150 mg every 8 h & \multirow{3}{*}{$\begin{array}{l}\text { Siriraj Hospital regimen } \\
\text { Nation, et al. regimen } \\
\text { EMA-approved regimen } \\
\text { FDA-approved regimen }\end{array}$} \\
\hline & $180 \mathrm{mg}$ every $12 \mathrm{~h}$ & \\
\hline & $150 \mathrm{mg}$ every $12 \mathrm{~h}$ & \\
\hline \multirow{4}{*}{51 to $79 \mathrm{~mL} / \mathrm{min}$} & & EMA-approved regimen \\
\hline & 150 mg every 12 h & Siriraj Hospital regimen \\
\hline & & Nation, et al. regimen \\
\hline & $114 \mathrm{mg}$ every $12 \mathrm{~h}$ & FDA-approved regimen \\
\hline \multirow{4}{*}{30 to $50 \mathrm{~mL} / \mathrm{min}$} & $125 \mathrm{mg}$ every $12 \mathrm{~h}$ & EMA-approved regimen \\
\hline & $110 \mathrm{mg}$ every $12 \mathrm{~h}$ & Nation, et al. regimen \\
\hline & $150 \mathrm{mg}$ every $24 \mathrm{~h}$ & FDA-approved regimen \\
\hline & $100 \mathrm{mg}$ every $12 \mathrm{~h}$ & Siriraj Hospital regimen \\
\hline \multirow{4}{*}{11 to $29 \mathrm{~mL} / \mathrm{min}$} & $180 \mathrm{mg}$ every $24 \mathrm{~h}$ & Nation, et al. regimen \\
\hline & $150 \mathrm{mg}$ every $24 \mathrm{~h}$ & EMA-approved regimen \\
\hline & & Siriraj Hospital regimen \\
\hline & $60 \mathrm{mg}$ every $24 \mathrm{~h}$ & FDA-approved regimen \\
\hline \multirow{3}{*}{$\leq 10 \mathrm{~mL} / \mathrm{min}$} & $150 \mathrm{mg}$ every $24 \mathrm{~h}$ & Nation, et al. regimen \\
\hline & 120 mg every $24 \mathrm{~h}$ & EMA-approved regimen \\
\hline & $60 \mathrm{mg}$ every $24 \mathrm{~h}$ & FDA-approved regimen \\
\hline
\end{tabular}


Table 3. Our study regimens for Simulations.

\begin{tabular}{|c|c|}
\hline Creatinine Clearance & Daily Dose (CBA) \\
\hline$\geq 80 \mathrm{~mL} / \mathrm{min}$ & 180 mg every $8 \mathrm{~h}$ \\
\hline 51 to $79 \mathrm{~mL} / \mathrm{min}$ & $\begin{array}{c}180 \mathrm{mg} \text { every } 8 \mathrm{~h} \\
150 \mathrm{mg} \text { every } 8 \mathrm{~h} \\
180 \mathrm{mg} \text { every } 12 \mathrm{~h} \\
100 \mathrm{mg} \text { every } 8 \mathrm{~h}\end{array}$ \\
\hline 30 to $50 \mathrm{~mL} / \mathrm{min}$ & $\begin{array}{c}180 \mathrm{mg} \text { every } 8 \mathrm{~h} \\
150 \mathrm{mg} \text { every } 8 \mathrm{~h} \\
180 \mathrm{mg} \text { every } 12 \mathrm{~h} \\
150 \mathrm{mg} \text { every } 12 \mathrm{~h} \\
100 \mathrm{mg} \text { every } 8 \mathrm{~h}\end{array}$ \\
\hline 11 to $29 \mathrm{~mL} / \mathrm{min}$ & $\begin{array}{c}180 \mathrm{mg} \text { every } 8 \mathrm{~h} \\
180 \mathrm{mg} \text { every } 12 \mathrm{~h} \\
150 \mathrm{mg} \text { every } 12 \mathrm{~h} \\
150 \mathrm{mg} \text { every } 8 \mathrm{~h}\end{array}$ \\
\hline$\leq 10 \mathrm{~mL} / \mathrm{min}$ & $\begin{array}{l}180 \mathrm{mg} \text { every } 8 \mathrm{~h} \\
150 \mathrm{mg} \text { every } 8 \mathrm{~h} \\
100 \mathrm{mg} \text { every } 8 \mathrm{~h} \\
180 \mathrm{mg} \text { every } 12 \mathrm{~h} \\
150 \mathrm{mg} \text { every } 12 \mathrm{~h} \\
100 \mathrm{mg} \text { every } 12 \mathrm{~h} \\
180 \mathrm{mg} \text { every } 24 \mathrm{~h}\end{array}$ \\
\hline
\end{tabular}

$\mathrm{CBA}=$ colistin base activity, EMA = European Medicine Agency, FDA = US-Food and Drug Administration. All of regimens were infused over $30 \mathrm{~min}$. The alternative data of Table 3 were shown in Supplementary Materials Table S2.

\subsection{Monte Carlo Simulation}

Monte Carlo Simulation (Crystal Ball version 2017; Decisioneering Inc., Denver, CO USA) was conducted to generate 10,000 subjects for IV dosage regimens of colistin based on the linear pharmacokinetic behavior. The $f$ AUC/MIC following multiple intravenous doses of various regimens was determined for the profile of each individual. Log-normal distributions were studied for between-patient variability. Probability of target attainment (PTA) was determined as the percentage of all 10,000 estimates which achieved or exceeded pharmacodynamics surrogate indices ( $f$ AUC/MIC 225 ) with increasing MIC [13]. The cumulative fraction of response (CFR) was calculated as the proportion of \%PTA of each MIC according to the MIC distribution. The PTA and CFR of $90 \%$ were considered successful.

\section{Results}

\subsection{Microbiology}

All clinical isolates of 116 CRE include carbapenem-resistant K. pneumoniae ( $\mathrm{n}=96)$ and carbapenem-resistant E. coli $(\mathrm{n}=20)$ were used. Colistin MIC's ranged from $0.25->128 \mathrm{mcg} / \mathrm{mL}$. The overall colistin $\mathrm{MIC}_{50}$ and $\mathrm{MIC}_{90}$ were 1 and $16 \mathrm{mcg} / \mathrm{mL}$, respectively. Among the carbapenem-resistant isolates, 91 of 116 isolates $(78.44 \%$ ) were susceptible to colistin (MIC $\leq 2 \mathrm{mcg} / \mathrm{mL}$ ). Of the colistin-susceptible isolates, 74 were K. pneumoniae $(74 / 91 ; 81.32 \%)$ and 17 were E. coli $(17 / 91$; $18.68 \%$ ). Colistin-susceptible K. pneumoniae isolates had $\mathrm{MIC}_{50}$ and $\mathrm{MIC}_{90}$ of 0.5 and $2 \mathrm{mcg} / \mathrm{mL}$, respectively. For colistin-susceptible $E$. coli isolates, they had $\mathrm{MIC}_{50}$ and $\mathrm{MIC}_{90}$ of 0.5 and $0.5 \mathrm{mcg} / \mathrm{mL}$, respectively. While, 25 of 116 isolates $(21.56 \%)$ were resistant to colistin, of which 22 were K. pneumoniae $(22 / 25 ; 88 \%)$ and 3 were E. coli $(3 / 25 ; 12 \%)$. Colistin-resistant K. pneumoniae isolates had $\mathrm{MIC}_{50}$ and $\mathrm{MIC}_{90}$ of 16 and $32 \mathrm{mcg} / \mathrm{mL}$, respectively. For colistin-resistant $E$. coli isolates, they had $\mathrm{MIC}_{50}$ and 
$\mathrm{MIC}_{90}$ of 8 and $32 \mathrm{mcg} / \mathrm{mL}$, respectively. Minimum inhibitory concentrations (MICs) values of the study isolates are shown in Table 4.

Table 4. Minimum inhibitory concentrations (MICs) distribution of colistin.

\begin{tabular}{|c|c|c|c|c|c|c|c|c|c|c|c|c|}
\hline $\operatorname{MIC}(\mathrm{mcg} / \mathrm{mL})$ & 0.25 & 0.5 & 1 & 2 & 4 & 8 & 16 & 32 & 64 & $>128$ & $\begin{array}{c}\mathrm{MIC}_{50} \\
(\mathrm{mcg} / \mathrm{mL})\end{array}$ & $\begin{array}{c}\mathrm{MIC}_{90} \\
(\mathrm{mcg} / \mathrm{mL})\end{array}$ \\
\hline $\begin{array}{c}\text { All isolates } \\
(n=116)\end{array}$ & 3 & 55 & 19 & 14 & 4 & 5 & 10 & 4 & 1 & 1 & 1 & 16 \\
\hline$\%$ & 2.58 & 47.41 & 16.38 & 12.07 & 3.45 & 4.31 & 8.6 & 3.45 & 0.86 & 0.86 & & \\
\hline \multicolumn{13}{|c|}{ Colistin-Susceptible Isolates (MIC $\leq 2 \mathrm{mcg} / \mathrm{mL}$ ) } \\
\hline $\begin{array}{c}K \cdot p \text { pneumoniae } \\
(\mathrm{n}=74)\end{array}$ & 3 & 40 & 18 & 13 & - & - & - & - & - & - & 0.5 & 2 \\
\hline$E . \operatorname{coli}(\mathrm{n}=17)$ & - & 15 & 1 & 1 & - & - & - & - & - & - & 0.5 & 0.5 \\
\hline \multicolumn{13}{|c|}{ Colistin-Resistant Isolates (MIC > $2 \mathrm{mcg} / \mathrm{mL}$ ) } \\
\hline $\begin{array}{c}\text { K. pneumoniae } \\
(\mathrm{n}=22)\end{array}$ & - & - & - & - & 3 & 4 & 10 & 3 & 1 & 1 & 16 & 32 \\
\hline$E . \operatorname{coli}(\mathrm{n}=3)$ & - & - & - & - & 1 & 1 & - & 1 & - & - & 8 & 32 \\
\hline
\end{tabular}

\subsection{Pharmacokinetic-Pharmacodynamic Simulations}

\subsubsection{Probability of Target Attainment}

The PTA analyses of various colistin regimens and creatinine clearance to achieve 90\% PTA against carbapenem-resistant K. pneumoniae and carbapenem-resistant $E$. coli following the $f$ AUC/MIC $\geq 25$ are shown in Figure 1A-E. This figures include the \%PTA for the EMA-, FDA-, Nation et al., Siriraj Hospital together with our study regimens. None of the colistin dosing regimens achieved PTA target against the $\mathrm{MIC}_{90}$ for colistin-resistant K. pneumoniae and colistin-resistant E. coli (32 mcg/mL) in all renal function groups. Similarly, none of the colistin dosing regimens achieved $\geq 90 \%$ PTA against the $\mathrm{MIC}_{50}$ for colistin-resistant K. pneumoniae $(16 \mathrm{mcg} / \mathrm{mL})$ in patients with creatinine clearance $>10 \mathrm{~mL} / \mathrm{min}$. For patients with creatinine clearance $\leq 10 \mathrm{~mL} / \mathrm{min}$, attainment rate of $90 \%$ was observed only in our study regimen using $180 \mathrm{mg}$ every $8 \mathrm{~h}$. At the $\mathrm{MIC}_{50}$ for colistin-resistant $E$. coli $(8 \mathrm{mcg} / \mathrm{mL})$, none of the colistin approved by EMA-, FDA-, Nation et al. as well as Siriraj Hospital achieved PTA target in all renal function groups. However, our study regimens with daily dose of at least $450 \mathrm{mg}$ at creatinine clearance $11-29 \mathrm{~mL} / \mathrm{min}$ and daily doses of at least $360 \mathrm{mg}$ at creatinine clearance $\leq 10 \mathrm{~mL} / \mathrm{min}$ could achieve $90 \%$ PTA.

At the MIC of $0.5 \mathrm{mcg} / \mathrm{mL}\left(\mathrm{MIC}_{50}\right.$ for colistin-susceptible $K$. pneumoniae and $\mathrm{MIC}_{50} / \mathrm{MIC}_{90}$ for colistin-susceptible E. coli), all of the dosage regimens achieved $\geq 90 \%$ PTA regardless of renal function. At the MIC of $2 \mathrm{mcg} / \mathrm{mL}$ ( $\mathrm{MIC}_{90}$ for colistin-susceptible K. pneumoniae and the current susceptibility breakpoint), none of the colistin dosing regimens achieved $\geq 90 \%$ PTA in patients with creatinine clearance $\geq 80 \mathrm{~mL} / \mathrm{min}$. However, the EMA-, FDA-, Nation et al. and Siriraj Hospital approved regimens provided PTA of 64.41, 69.2 and 79.7\%, respectively. For patients with creatinine clearance 11-79 mL/min, the EMA-, FDA-, Nation et al. as well as Siriraj Hospital provided PTA of 55-89\%. However, our study regimens using daily doses of $540 \mathrm{mg}$ at creatinine clearance $51-79 \mathrm{~mL} / \mathrm{min}$ and daily doses of at least $300 \mathrm{mg}$ at creatinine clearance $11-50 \mathrm{~mL} / \mathrm{min}$ could provide an attainment rate $>90 \%$. Finally, in patients with creatinine clearance $\leq 10 \mathrm{~mL} / \mathrm{min}$, an attainment rate of $90 \%$ PTA was observed for all dosage regimens with daily doses of at least $120 \mathrm{mg}$ except the FDA-approved regimens.

The recommended dose based on the ability to achieve PTA target at various MICs with each dosing regimen are shown in Tables 5 and 6.

\subsubsection{Cumulative Fraction of Response}

Tables 7 and 8 summarizes the cumulative fraction of response (CFR) for colistin against 116 carbapenem-resistant K. pneumoniae and carbapenem-resistant E. coli. Overall, there was lower than $90 \%$ 
CFR for all of regimens in patients with creatinine clearance $>50 \mathrm{~mL} / \mathrm{min}$. For patients with creatinine clearance $\leq 50 \mathrm{~mL} / \mathrm{min}$, higher doses of at least $450 \mathrm{mg}$ per day in patients with creatinine clearance 30-50 $\mathrm{mL} / \mathrm{min}$, at least $300 \mathrm{mg}$ per day in patients with creatinine clearance $11-29 \mathrm{~mL} / \mathrm{min}$ and at least $200 \mathrm{mg}$ per day in patients with creatinine clearance $\leq 10 \mathrm{~mL} / \mathrm{min}$ could achieve the CFR target. For colistin-resistant isolates subgroup, the currently approved regimens achieved CFR target in only 10-50\%.

The CFR for colistin against colistin-susceptible $K$. pneumoniae and colistin-susceptible E. coli subgroup are shown in Tables 7 and 8. For colistin-susceptible K. pneumoniae, patients with creatinine clearance $\geq 80 \mathrm{~mL} / \mathrm{min}$, attainment rate of $90 \%$ was observed only in Siriraj Hospital and our study regimens (150 mg every $8 \mathrm{~h}$ and $180 \mathrm{mg}$ every $8 \mathrm{~h}$, respectively). While, attainment rates were higher than $90 \%$ with all dosage regimens in patients with creatinine clearance $30-50$ and $\leq 10 \mathrm{~mL} / \mathrm{min}$. For patients with creatinine clearance 51-79 and 11-29 mL/min, all dosage regimens achieved the CFR target except the FDA-approved regimens. In contrast, for colistin-susceptible E. coli, all dosage regimens achieved $\geq 90 \%$ CFR regardless of renal function.

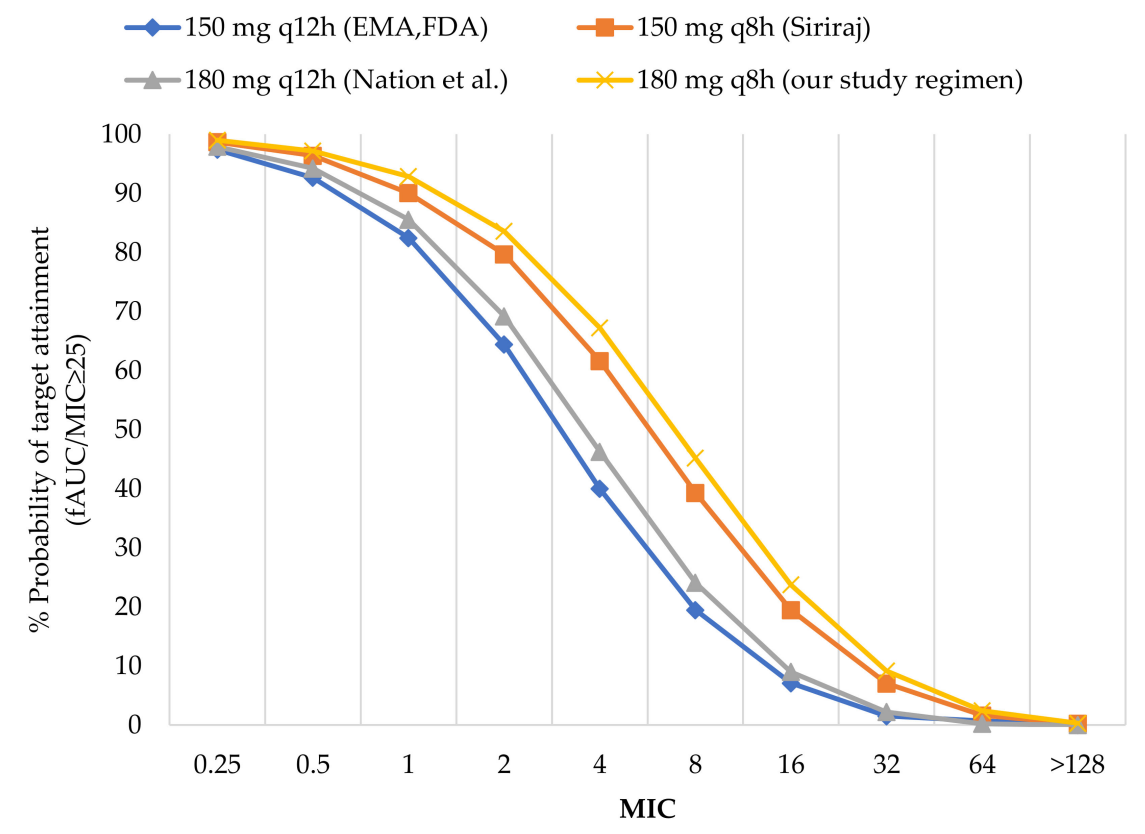

(A)

Figure 1. Cont. 


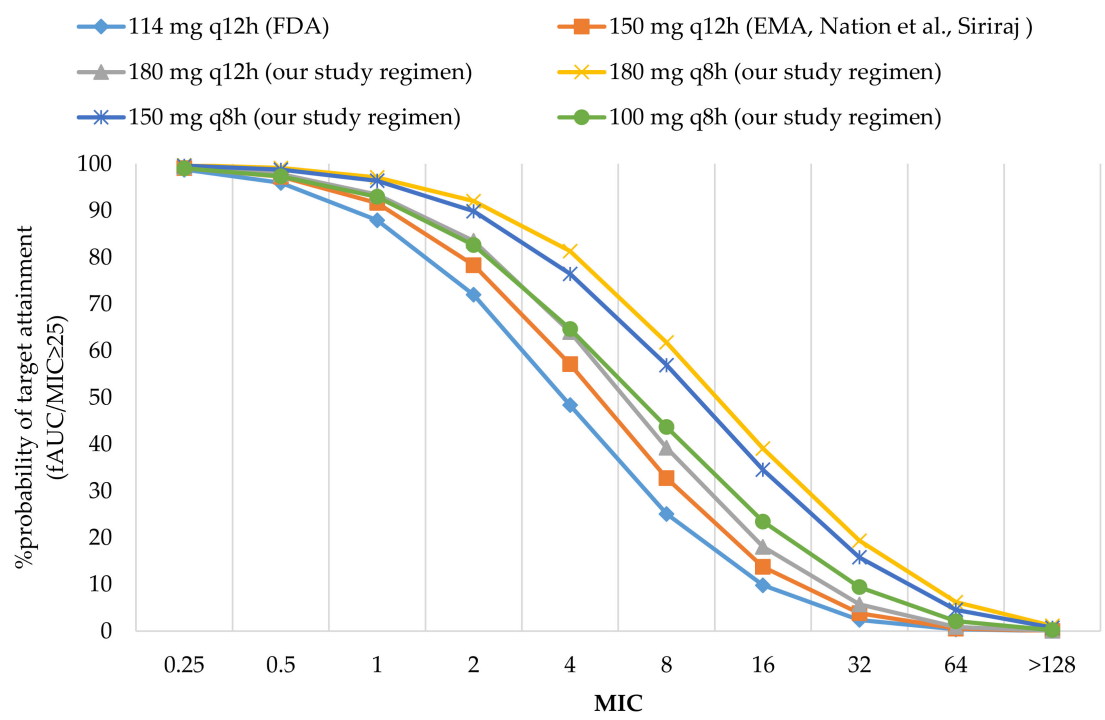

(B)

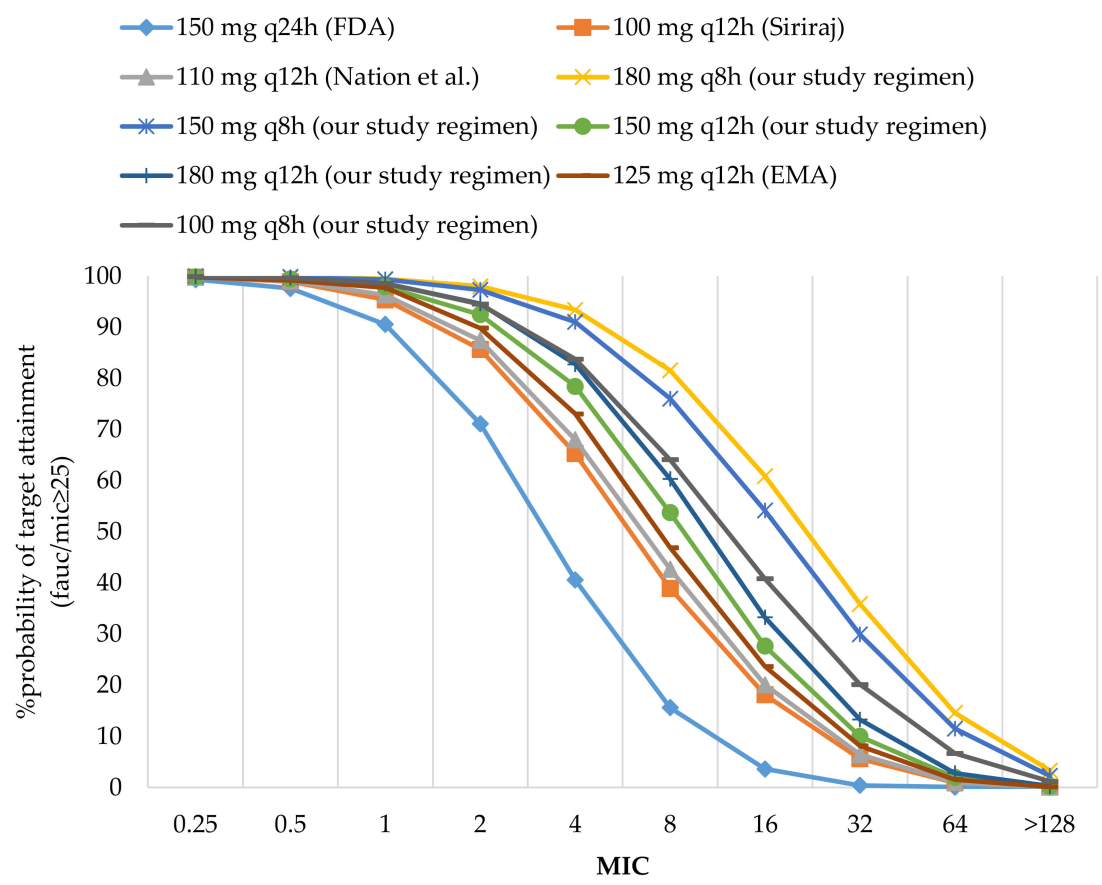

(C)

Figure 1. Cont. 


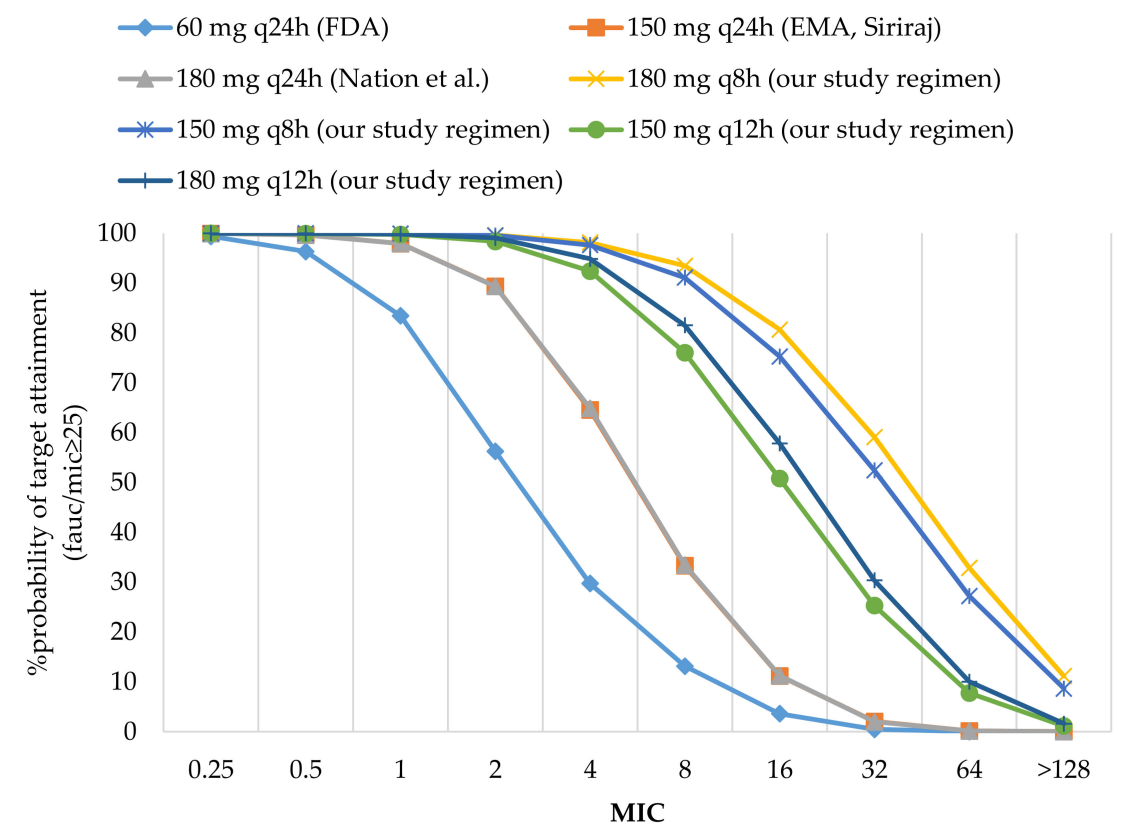

(D)

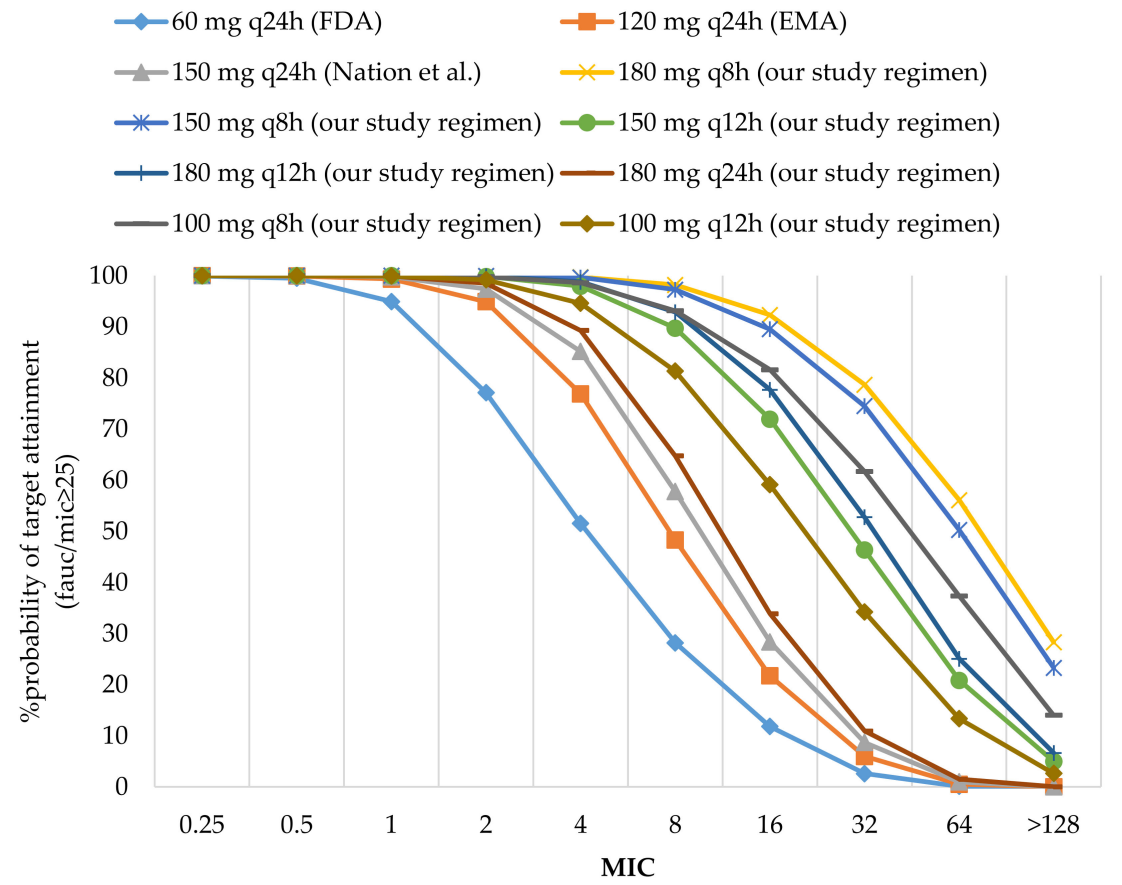

(E)

Figure 1. (A) \%PTA of colistin in patients with $\mathrm{CrCl} \geq 80$ (mL/min). (B) \%PTA of colistin in patients with $\mathrm{CrCl} 51-79$ (mL/min). (C) \%PTA of colistin in patients with $\mathrm{CrCl} 30-50$ (mL/min). (D) \%PTA of colistin in patients with $\mathrm{CrCl} 11-29$ (mL/min). (E) \%PTA of colistin in patients with $\mathrm{CrCl} \leq 10(\mathrm{~mL} / \mathrm{min})$. The alternative data of Figure (D) and (E) were shown in Supplementary Materials Figure S1 and S2. 
Table 5. The recommended dose based on the ability to achieve PTA target at various MICs.

\begin{tabular}{|c|c|c|c|}
\hline $\begin{array}{l}\text { Creatinine Clearance } \\
\qquad(\mathrm{mL} / \mathrm{min})\end{array}$ & $\begin{array}{c}\text { MIC } 0.5 \mathrm{mcg} / \mathrm{mL} \\
\text { Daily Dose (CBA) }\end{array}$ & $\begin{array}{c}\text { MIC } 2 \mathrm{mcg} / \mathrm{mL} \\
\text { Daily Dose (CBA) }\end{array}$ & $\begin{array}{c}\text { MIC } 8 \mathrm{mcg} / \mathrm{mL} \\
\text { Daily Dose (CBA) }\end{array}$ \\
\hline$\geq 80$ & $\begin{array}{l}150 \text { mg every } 12 \mathrm{~h} \\
\text { (EMA, FDA) }\end{array}$ & Not recommended & Not recommended \\
\hline $51-79$ & $\begin{array}{l}114 \text { mg every } 12 \mathrm{~h} \\
\text { (FDA) }\end{array}$ & $\begin{array}{l}180 \text { mg every } 8 \mathrm{~h} \\
\text { (our study) }\end{array}$ & Not recommended \\
\hline $30-50$ & $\begin{array}{c}150 \text { mg every } 24 \mathrm{~h} \\
\text { (FDA) }\end{array}$ & $\begin{array}{l}150 \text { mg every } 12 \mathrm{~h} \\
\text { (our study) }\end{array}$ & Not recommended \\
\hline $11-29$ & $\begin{array}{l}60 \text { mg every } 24 \mathrm{~h} \\
\text { (FDA) }\end{array}$ & $\begin{array}{l}150 \text { mg every } 12 \text { h } \\
\text { (our study) }\end{array}$ & $\begin{array}{l}150 \text { mg every } 8 \mathrm{~h} \\
\text { (our study) }\end{array}$ \\
\hline$\leq 10$ & $\begin{array}{l}60 \mathrm{mg} \text { every } 24 \mathrm{~h} \\
\text { (FDA) }\end{array}$ & $\begin{array}{c}120 \text { mg every } 24 \mathrm{~h} \\
\text { (EMA) }\end{array}$ & $\begin{array}{l}180 \text { mg every } 12 \mathrm{~h} \\
\text { (our study) }\end{array}$ \\
\hline
\end{tabular}

The alternative data of Table 5 were shown in Supplementary Materials Table S4.

Table 6. The recommended dose based on the ability to achieve PTA target at various MICs.

\begin{tabular}{ccc}
\hline $\begin{array}{c}\text { Creatinine Clearance } \\
(\mathrm{mL} / \mathrm{min})\end{array}$ & $\begin{array}{c}\text { MIC } 16 \mathrm{mcg} / \mathrm{mL} \\
\text { Daily Dose }(\mathrm{CBA})\end{array}$ & $\begin{array}{c}\text { MIC } 32 \mathrm{mcg} / \mathrm{mL} \\
\text { Daily Dose (CBA) }\end{array}$ \\
\hline $\mathbf{8 0 0}$ & Not recommended & Not recommended \\
$\mathbf{5 1 - 7 9}$ & Not recommended & Not recommended \\
$\mathbf{3 0 - 5 0}$ & Not recommended & Not recommended \\
$\mathbf{1 1 - 2 9}$ & $\begin{array}{c}\text { Not recommended } \\
180 \text { mg every } 8 \mathrm{~h}\end{array}$ & Not recommended \\
$\leq \mathbf{1 0}$ & (our study) & Not recommended \\
\hline
\end{tabular}

MICs = minimum inhibitory concentrations $(\mathrm{mcg} / \mathrm{mL})$, EMA = European Medicine Agency, FDA = US-Food and Drug Administration, CBA = colistin base activity. The alternative data of Table 6 were shown in Supplementary Materials Table S5. 
Table 7. \%PTA and \%CFR for All isolates, Colistin-susceptible isolates subgroup and Colistin-resistant isolates subgroup.

\begin{tabular}{|c|c|c|c|c|c|c|c|c|c|c|c|c|c|c|c|c|c|}
\hline \multirow{5}{*}{$\mathrm{MIC}(\mathrm{mcg} / \mathrm{mL})$} & \multicolumn{3}{|c|}{ All Isolates } & \multicolumn{7}{|c|}{ Colistin-Susceptible Isolates } & \multicolumn{7}{|c|}{ Colistin-Resistant Isolates } \\
\hline & & & \multirow{4}{*}{$\%$ CFR } & \multicolumn{3}{|c|}{$\begin{array}{l}\text { Colistin-Susceptible } \\
\text { K. pneumoniae } \\
\text { Subgroup }\end{array}$} & \multicolumn{3}{|c|}{$\begin{array}{l}\text { Colistin-Susceptible } \\
\text { E. coli Subgroup }\end{array}$} & \multirow{4}{*}{$\begin{array}{l}\text { \%CFR for all } \\
\text { Sus-Ceptible } \\
\text { Isolates }\end{array}$} & \multicolumn{3}{|c|}{$\begin{array}{l}\text { Colistin-Resistant } \\
\text { K. pneumoniae Subgroup }\end{array}$} & \multicolumn{3}{|c|}{$\begin{array}{l}\text { Colistin-Resistant } \\
\text { E. coli Subgroup }\end{array}$} & \multirow{4}{*}{$\begin{array}{c}\text { \%CFR for al } \\
\text { Resistant } \\
\text { Isolates }\end{array}$} \\
\hline & \multicolumn{2}{|c|}{$\%$ PTA } & & \multicolumn{2}{|c|}{$\%$ PTA } & \multirow{3}{*}{$\%$ CFR } & \multicolumn{2}{|c|}{$\%$ PTA } & \multirow{3}{*}{$\%$ CFR } & & \multicolumn{2}{|c|}{ \%PTA } & \multirow{3}{*}{$\%$ CFR } & \multicolumn{2}{|c|}{$\%$ PTA } & \multirow{3}{*}{$\%$ CFR } & \\
\hline & $\mathrm{MIC}_{50}$ & $\mathrm{MIC}_{90}$ & & $\mathrm{MIC}_{50}$ & $\mathrm{MIC}_{90}$ & & $\mathrm{MIC}_{50}$ & $\mathrm{MIC}_{90}$ & & & $\mathrm{MIC}_{50}$ & $\mathrm{MIC}_{90}$ & & $\mathrm{MIC}_{50}$ & $\mathrm{MIC}_{90}$ & & \\
\hline & 1 & 16 & & 0.5 & 2 & & 0.5 & 0.5 & & & 16 & 32 & & 8 & 32 & & \\
\hline \multicolumn{18}{|l|}{$\mathrm{CrCl} \geq 80 \mathrm{~mL} / \mathrm{min}$} \\
\hline $\begin{array}{l}150 \mathrm{mg} \text { q12 h } \\
\text { (EMA, FDA) }\end{array}$ & 82.47 & 7.08 & 70.68 & 92.66 & 64.41 & 85.41 & 92.66 & 92.66 & 90.40 & 86.34 & 7.08 & 1.51 & 12.45 & 19.48 & 1.51 & 20.33 & 13.40 \\
\hline $\begin{array}{l}150 \mathrm{mg} \mathrm{q} 8 \mathrm{~h} \\
\text { (Siriraj) }\end{array}$ & 90.07 & 19.48 & 78.56 & 98.68 & 79.7 & 92.02 & 98.68 & 98.68 & 95.06 & 92.58 & 19.48 & 7.02 & 25.45 & 39.3 & 7.02 & 35.99 & 26.71 \\
\hline \multicolumn{18}{|l|}{$\mathrm{CrCl} 51$ to $79 \mathrm{~mL} / \mathrm{min}$} \\
\hline $\begin{array}{c}114 \mathrm{mg} \mathrm{q} 12 \mathrm{~h} \\
\text { (FDA) }\end{array}$ & 87.96 & 9.8 & 74.90 & 95.95 & 71.98 & 89.91 & 95.95 & 95.95 & 94.07 & 90.69 & 9.8 & 2.36 & 15.94 & 25.05 & 2.36 & 25.26 & 17.06 \\
\hline $\begin{array}{l}150 \mathrm{mg} \mathrm{q} 12 \mathrm{~h} \\
\text { (Siriraj, EMA, } \\
\text { Nation et al) }\end{array}$ & 91.66 & 13.73 & 77.96 & 97.26 & 78.32 & 92.65 & 97.26 & 97.26 & 95.82 & 93.24 & 13.73 & 3.8 & 20.53 & 32.72 & 3.8 & 31.22 & 21.81 \\
\hline \multicolumn{18}{|l|}{$\mathrm{CrCl} 30$ to $50 \mathrm{~mL} / \mathrm{min}$} \\
\hline 150 mg q24 h (FDA) & 90.53 & 3.58 & 74.65 & 97.55 & 71.1 & $91 . .27$ & 97.55 & 97.55 & 95.58 & 92.07 & 3.58 & 0.39 & 10.05 & 15.59 & 0.39 & 18.84 & 11.10 \\
\hline $\begin{array}{l}110 \mathrm{mg} \mathrm{q} 12 \mathrm{~h} \\
\text { (Nation et al.) }\end{array}$ & 96.29 & 20.03 & 82.17 & 99.03 & 87.43 & 96.35 & 99.03 & 99.03 & 98.19 & 96.70 & 20.03 & 6.41 & 27.05 & 42.66 & 6.41 & 39.01 & 28.48 \\
\hline 125 mg q12 h (EMA) & 97.71 & 23.65 & 83.51 & 99.15 & 89.8 & 97.18 & 99.15 & 99.15 & 98.52 & 97.43 & 23.65 & 8.09 & 30.41 & 46.87 & 8.09 & 42.66 & 31.87 \\
\hline \multicolumn{17}{|l|}{$\mathrm{CrCl} 11$ to $29 \mathrm{~mL} / \mathrm{min}$} & 8.87 \\
\hline $\begin{array}{l}150 \mathrm{mg} \mathrm{q} 24 \mathrm{~h} \\
\text { (EMA, Siriraj) }\end{array}$ & 97.92 & 11.16 & 81.45 & 99.67 & 89.35 & 97.44 & 99.67 & 99.67 & 99.61 & 97.73 & 11.16 & 2.02 & 20.20 & 33.24 & 2.02 & 33.27 & 21.77 \\
\hline $\begin{array}{l}180 \mathrm{mg} \mathrm{q} 24 \mathrm{~h} \\
\text { (Nation et al.) }\end{array}$ & 97.93 & 11.23 & 81.45 & 99.63 & 89.32 & 97.41 & 99.63 & 99.63 & 99.93 & 97.70 & 11.23 & 1.94 & 20.29 & 33.41 & 1.94 & 33.4 & 21.87 \\
\hline $\begin{array}{l}\mathrm{CrCl} \leq 10 \mathrm{~mL} / \mathrm{min} \\
60 \mathrm{mg} \mathrm{g} 24 \mathrm{~h}(\mathrm{FDA})\end{array}$ & 94.94 & 11.81 & 78.82 & 99.5 & 77.09 & 94.47 & 99.5 & 99.5 & 99.99 & 95.11 & 11.81 & 2.61 & 17.88 & 28.19 & 2.61 & 27.44 & 19.03 \\
\hline $120 \mathrm{mg}$ q24 h (EMA) & 99.34 & 21.74 & 84.70 & 99.94 & 94.92 & 98.91 & 99.94 & 99.94 & 99.99 & 99.04 & 21.74 & 5.98 & 29.99 & 48.29 & 5.98 & 43.72 & 31.63 \\
\hline $\begin{array}{l}150 \mathrm{mg} \mathrm{q} 24 \mathrm{~h} \\
\text { (Nation et al.) }\end{array}$ & 99.76 & 28.36 & 86.51 & 99.98 & 97.4 & 99.47 & 99.98 & 99.98 & 100 & 99.54 & 28.36 & 8.69 & 36.24 & 57.76 & 8.69 & 50.55 & 37.96 \\
\hline
\end{tabular}

$\mathrm{MICs}=$ minimum inhibitory concentrations $(\mathrm{mcg} / \mathrm{mL}), \mathrm{PTA}=$ Probability of target attainment, $\mathrm{CFR}=\mathrm{Cumulative}$ fraction of response, $\mathrm{CrCl}=\mathrm{Creatinine}$ clearance $(\mathrm{ml} / \mathrm{min}), \mathrm{EMA}=\mathrm{European}$ Medicine Agency, FDA = US-Food and Drug Administration. The alternative data of Table 7 were shown in Supplementary Materials Table S6. 
Table 8. \%PTA and \%CFR of our study regimens for All isolates, Colistin-susceptible isolates subgroup and Colistin-resistant isolates subgroup.

\begin{tabular}{|c|c|c|c|c|c|c|c|c|c|c|c|c|c|c|c|c|c|}
\hline \multirow{5}{*}{$\mathrm{MIC}(\mathrm{mcg} / \mathrm{mL})$} & \multicolumn{3}{|c|}{ All Isolates } & \multicolumn{7}{|c|}{ Colistin-Susceptible Isolates } & \multicolumn{7}{|c|}{ Colistin-Resistant Isolates } \\
\hline & & & \multirow{4}{*}{$\%$ CFR } & \multirow{2}{*}{\multicolumn{3}{|c|}{$\begin{array}{c}\text { Colistin-Susceptible } \\
\text { K. pneumoniae Subgroup } \\
\end{array}$}} & \multicolumn{3}{|c|}{$\begin{array}{l}\text { Colistin-Susceptible } \\
\text { E. coli Subgroup }\end{array}$} & \multirow{4}{*}{$\begin{array}{l}\text { \%CFR for all } \\
\text { Sus-Ceptible } \\
\text { Isolates }\end{array}$} & \multirow{2}{*}{\multicolumn{3}{|c|}{$\begin{array}{c}\begin{array}{c}\text { Colistin-Resistant } \\
\text { K. pneumoniae Subgroup }\end{array} \\
\% \text { PTA }\end{array}$}} & \multicolumn{3}{|c|}{$\begin{array}{c}\text { Colistin-Resistant E. coli } \\
\text { Subgroup }\end{array}$} & \multirow{4}{*}{$\begin{array}{c}\text { \%CFR for al } \\
\text { Resistant } \\
\text { Isolates }\end{array}$} \\
\hline & \multicolumn{2}{|c|}{$\%$ PTA } & & & & & \multicolumn{2}{|c|}{$\%$ PTA } & \multirow{3}{*}{$\%$ CFR } & & & & \multirow{3}{*}{$\%$ CFR } & \multicolumn{2}{|c|}{$\%$ PTA } & \multirow{3}{*}{$\%$ CFR } & \\
\hline & $\mathrm{MIC}_{50}$ & $\mathrm{MIC}_{90}$ & & $\mathrm{MIC}_{50}$ & $\mathrm{MIC}_{90}$ & \multirow{2}{*}{$\%$ CFR } & $\mathrm{MIC}_{50}$ & $\mathrm{MIC}_{90}$ & & & $\mathrm{MIC}_{50}$ & $\mathrm{MIC}_{90}$ & & $\mathrm{MIC}_{50}$ & $\mathrm{MIC}_{90}$ & & \\
\hline & 1 & 16 & & 0.5 & 2 & & 0.5 & 0.5 & & & 16 & 32 & & 8 & 32 & & \\
\hline \multicolumn{18}{|l|}{$\mathrm{CrCl} \geq 80 \mathrm{~mL} / \mathrm{min}$} \\
\hline $180 \mathrm{mg} \mathrm{q} 8 \mathrm{~h}$ & 92.88 & 23.74 & 80.80 & 97.19 & 83.61 & 93.83 & 97.19 & 97.19 & 96.14 & 92.58 & 23.74 & 9.17 & 29.55 & 45.2 & 9.17 & 40.54 & 30.87 \\
\hline \multicolumn{18}{|l|}{$\mathrm{CrCl} 51$ to $79 \mathrm{~mL} / \mathrm{min}$} \\
\hline $100 \mathrm{mg} \mathrm{q} 8 \mathrm{~h}$ & 92.96 & 23.43 & 80.60 & 97.36 & 82.62 & 93.77 & 97.36 & 97.36 & 96.23 & 94.23 & 23.43 & 9.4 & 28.79 & 43.67 & 9.4 & 39.25 & 30.05 \\
\hline 150 mg q8 h & 96.39 & 34.52 & 85.01 & 98.79 & 89.88 & 96.68 & 98.79 & 98.79 & 98.12 & 96.94 & 34.52 & 15.78 & 38.86 & 56.93 & 15.78 & 49.72 & 40.16 \\
\hline $180 \mathrm{mg} \mathrm{q} 8 \mathrm{~h}$ & 97.13 & 39.07 & 86.51 & 99.15 & 92.05 & 97.44 & 99.15 & 99.15 & 98.61 & 97.65 & 39.07 & 19.36 & 43.05 & 61.77 & 19.36 & 54.16 & 44.38 \\
\hline \multicolumn{18}{|l|}{$\mathrm{CrCl} 30$ to $50 \mathrm{~mL} / \mathrm{min}$} \\
\hline $100 \mathrm{mg} \mathrm{q} 8 \mathrm{~h}$ & 98.54 & 40.8 & 87.65 & 99.64 & 94.49 & 98.48 & 99.64 & 99.64 & 99.27 & 98.63 & 40.8 & 20.09 & 44.71 & 64.06 & 20.09 & 55.97 & 46.06 \\
\hline $150 \mathrm{mg} \mathrm{q} 12 \mathrm{~h}$ & 97.9 & 27.61 & 84.91 & 99.41 & 92.44 & 97.84 & 99.41 & 99.41 & 98.91 & 98.04 & 27.61 & $9 . .97$ & 34.47 & 53.71 & 9.97 & 47.36 & 36.01 \\
\hline $180 \mathrm{mg} \mathrm{q} 12 \mathrm{~h}$ & 98.52 & 33.25 & 86.48 & 99.67 & 94.6 & 98.51 & 99.67 & 99.67 & 99.30 & 98.66 & 33.25 & 13.25 & 39.30 & 60.3 & 13.25 & 52.09 & 40.84 \\
\hline 150 mg q8 h & 99.33 & 54.13 & 90.59 & 99.76 & 97.29 & 99.23 & 99.76 & 99.76 & 99.59 & 99.30 & 54.13 & 29.89 & 55.53 & 76.01 & 29.89 & 65.64 & 56.75 \\
\hline $180 \mathrm{mg} \mathrm{q} 8 \mathrm{~h}$ & 99.41 & 60.87 & 91.96 & 99.9 & 97.97 & 99.44 & 99.9 & 99.9 & 99.76 & 99.50 & 60.87 & 35.86 & 60.93 & 81.54 & 35.86 & 70.26 & 62.05 \\
\hline \multicolumn{18}{|l|}{$\mathrm{CrCl} 11$ to $29 \mathrm{~mL} / \mathrm{min}$} \\
\hline $150 \mathrm{mg} \mathrm{q} 12 \mathrm{~h}$ & 99.76 & 50.76 & 90.39 & 99.91 & 98.35 & 99.60 & 99.91 & 99.91 & 99.81 & 99.64 & 50.76 & 25.25 & 53.34 & 76.03 & 25.25 & 64.55 & 54.68 \\
\hline $180 \mathrm{mg}$ q $8 \mathrm{~h}$ & 99.93 & 80.65 & 95.87 & 100 & 99.63 & 99.92 & 100 & 100 & 99.97 & 99.93 & 80.65 & 59.11 & 77.09 & 93.47 & 59.11 & 83.57 & 77.87 \\
\hline \multicolumn{18}{|l|}{$\mathrm{CrCl} \leq 10 \mathrm{~mL} / \mathrm{min}$} \\
\hline $180 \mathrm{mg} \mathrm{q} 24 \mathrm{~h}$ & 99.89 & 33.88 & 87.73 & 100 & 98.53 & 99.72 & 100 & 100 & 99.91 & 99.75 & 33.88 & 10.94 & 40.91 & 64.74 & 10.94 & 54.99 & 42.60 \\
\hline $100 \mathrm{mg} \mathrm{q} 12 \mathrm{~h}$ & 99.93 & 59.11 & 92.02 & 99.98 & 99.18 & 99.83 & 99.98 & 99.98 & 99.93 & 99.85 & 59.11 & 34.22 & 59.95 & 81.32 & 34.22 & 70.5 & 61.16 \\
\hline 100 mg q8 h & 99.99 & 81.59 & 96.14 & 100 & 99.79 & 99.96 & 100 & 100 & 99.99 & 99.96 & 81.59 & 61.67 & 78.21 & 93.13 & 61.67 & 84.47 & 78.96 \\
\hline $150 \mathrm{mg}$ q12 h & 99.98 & 71.94 & 94.13 & 100 & 99.84 & 99.98 & 100 & 100 & 99.99 & 99.97 & 71.94 & 46.32 & 69.86 & 89.74 & 46.32 & 78.00 & 70.84 \\
\hline $180 \mathrm{mg} \mathrm{q} 12 \mathrm{~h}$ & 99.98 & 77.7 & 95.30 & 100 & 99.90 & 99.97 & 100 & 100 & 99.99 & 99.98 & 77.7 & 52.76 & 74.31 & 92.85 & 52.76 & 81.47 & 75.17 \\
\hline 150 mg q8 h & 99.99 & 89.51 & 97.76 & 100 & 99.97 & 99.99 & 100 & 100 & 100 & 99.99 & 89.51 & 74.47 & 85.46 & 97.26 & 74.47 & 90.46 & 86.06 \\
\hline $180 \mathrm{mg} \mathrm{q} 8 \mathrm{~h}$ & 99.99 & 92.31 & 98.30 & 100 & 99.94 & 99.99 & 100 & 100 & 100 & 99.99 & 92.31 & 78.66 & 87.97 & 98.18 & 78.66 & 92.19 & 88.48 \\
\hline
\end{tabular}

$\mathrm{MICs}=$ minimum inhibitory concentrations $(\mathrm{mcg} / \mathrm{mL}), \mathrm{PTA}=$ Probability of target attainment, $\mathrm{CFR}=$ Cumulative fraction of response, $\mathrm{CrCl}=\mathrm{Creatinine}$ clearance $(\mathrm{mL} / \mathrm{min})$. The alternative data of Table 8 were shown in Supplementary Materials Table S7. 


\section{Discussion}

In our study, the MICs of colistin for CRE isolates were within the range of $0.25->128 \mathrm{mcg} / \mathrm{mL}$. The overall colistin $\mathrm{MIC}_{50}$ and $\mathrm{MIC}_{90}$ were 1 and $16 \mathrm{mcg} / \mathrm{mL}$, respectively. Our study showed $\mathrm{MIC}_{50} / \mathrm{MIC}_{90}$ of $1 / 16$ and $0.5 / 4 \mathrm{mcg} / \mathrm{mL}$ for carbapenem-resistant $K$. pneumoniae and carbapenem-resistant E. coli, respectively. In Thailand, a study which was performed at Siriraj Hospital [19] demonstrated $\mathrm{MIC}_{50} / \mathrm{MIC}_{90}$ of $32 />128$ and $0.5 / 8 \mathrm{mcg} / \mathrm{mL}$ for carbapenem-resistant $\mathrm{K}$. pneumoniae and carbapenem-resistant E. coli, respectively. A Global Surveillance Program study (including isolates of Enterobacteriaceae in Thailand) [20] demonstrated values of $\mathrm{MIC}_{50}$ and $\mathrm{MIC}_{90}$ at $\leq 0.012$ and $\geq 4$ $\mathrm{mcg} / \mathrm{mL}$, respectively. The discrepancies in MIC results among the studies could be due to the difference in prevalence of colistin resistance isolates $(21.56 \%$ in our study, $71.3 \%$ in study at Siriraj Hospital [19] and $<10 \%$ Global Surveillance Program study [20], respectively) as well as the percentage of K. pneumoniae isolates (82.75\% in our study, $88.32 \%$ in study at Siriraj Hospital [19] and <50\% Global Surveillance Program study [20], respectively). Our study showed the frequency of colistin resistance in K. pneumoniae to be higher than the frequency of colistin resistance in E. coli (22.9\% versus $15 \%$, respectively). In addition, the $\mathrm{MIC}_{50}$ and $\mathrm{MIC}_{90}$ values of $\mathrm{K}$. pneumoniae isolates were higher than E. coli isolates. This was consistent with previous studies which described the use of high MICs for colistin in K. pneumoniae $[19,21]$

This is the first study using Monte carlo simulation to determine effective dosage regimens for colistin against carbapenem-resistant K. pneumoniae and carbapenem-resistant $E$. coli. When colistin was administered as monotherapy, all of colistin regimens approved by EMA-, FDA-, Nation et al., Siriraj Hospital would not be effective against $\mathrm{MIC}_{90}$ of colistin-resistant K. pneumoniae and colistin-resistant E. coli $(32 \mathrm{mcg} / \mathrm{mL}), \mathrm{MIC}_{50}$ of colistin-resistant $K$. pneumoniae $(16 \mathrm{mcg} / \mathrm{mL})$ and $\mathrm{MIC}_{50}$ of colistin-resistant E. coli isolates $(8 \mathrm{mcg} / \mathrm{mL})$ as shown by the PTA falling below $90 \%$.

For isolates with $\mathrm{MIC} \leq 0.5 \mathrm{mcg} / \mathrm{mL}$ ( $\mathrm{MIC}_{50}$ for colistin-susceptible K. pneumoniae and $\mathrm{MIC}_{50} / \mathrm{MIC}_{90}$ for colistin-susceptible E. coli), all dosage regimens achieved $\geq 90 \%$ PTA regardless of renal function. This was consistent with a study by Tsala, et al. [13] which found $>95 \%$ PTA to be effective against K. pneumoniae when the MIC were $\leq 0.5 \mathrm{mcg} / \mathrm{mL}$ by using colistin $300 \mathrm{mg}$ CBA per day in patients with normal renal function. In contrast, there were lower success rates report with colistin against isolates with MIC of $2 \mathrm{mcg} / \mathrm{mL}$ ( $\mathrm{MIC}_{90}$ for colistin-susceptible K. pneumoniae and the current susceptibility breakpoint) using all of recommendations in patients who had creatinine clearance $>10 \mathrm{~mL} / \mathrm{min}$. However, the EMA-, FDA-, Nation et al. and Siriraj Hospital approved regimens achived PTA target in approximately $65-89 \%$. Interestingly, all the regimens with daily doses of at least $120 \mathrm{mg}$ could achieve the target for patients with creatinine clearance of $\leq 10 \mathrm{~mL} / \mathrm{min}$. This was consistent with a study by Tsala, et al. [13] which showed lower success rates using colistin $300 \mathrm{mg}$ CBA per day in patients who had normal renal function for isolates with $\mathrm{MIC} \geq 2 \mathrm{mcg} / \mathrm{mL}$.

Previous studied have shown that the EMA-, FDA- and Nation et al. approved regimens could achieve PTA target for patients with creatinine clearance $<80 \mathrm{~mL} / \mathrm{min}$. Our results were in contrast to the above studies, and these results might be explained because previous studies used the average plasma concentration at steady state (Css, avg) of $2 \mathrm{mcg} / \mathrm{mL}$ which was a reasonable PK/PD target of colistin against gram negative organisms [12,16]. However, the PK/PD target of Css, avg of $2 \mathrm{mcg} / \mathrm{mL}$ was appropriate for pathogen with $\mathrm{MIC} \leq 2 \mathrm{mcg} / \mathrm{mL}[12,16]$. The prevalence of colistin resistance (MIC $>2 \mathrm{mcg} / \mathrm{mL}$ ) among isolates in our study was rather high (approximately 40\%) and the MIC50 and MIC90 values were higher than other studies. For this reason, the PK/PD target of Css, avg of $2 \mathrm{mcg} / \mathrm{mL}$ was inappropriate to use in our study. The PK/PD targets $f$ AUC/MIC $\geq 25$, which were used in this study, were determined using limited information [13]. However, several studies including in vitro studies as well as infection models which used neutropenic mice have confirmed the relation to clinical applications with these targets for colistin $[13,14]$. In addition, our study used higher PTA target compared with other studies ( $\geq 90 \%$ versus $80-90 \%$ ).

The FDA-approved regimens have shown lower target attainment rates than other regimens. Similarly, the studies from Nation et al. [12,16] showed that FDA-approved regimens were substaintially 
lower than those approved by the EMA. The data has also shown that patients with renal impairment have a better chance to achieve PTA target compared with normal renal function. The findings of our study were consistent with previous analyses, the dosing suggestions of Nation et al. achieved $80-90 \%$ of patients in each of 3 groups of creatinine clearance patients (50 to $<80,30$ to $<50,<30 \mathrm{~mL} / \mathrm{min}$ ). At creatinine clearance $\geq 80 \mathrm{~mL} / \mathrm{min}$, the suggested doses only provided $<40 \%$ PTA for plasma colistin Css, avg of $2 \mathrm{mcg} / \mathrm{mL}$ [12]. Additionally, renal function significantly impacts upon the proportion of colistimethate for conversion to colistin due to colistimethate being predominately renally excreted by with only small fraction of the dose converted to colistin [9,22,23].

Our results have suggested that current maintenance doses may not be effective against isolates with an MIC $>0.5 \mathrm{mcg} / \mathrm{mL}$. This is consistent with a study by Garonsik, et al. [9] and Tsala, et al. [13] which showed this MIC as the highest MIC offering reasonable PTA for current maintenance doses. Similarly, previous studies have reported that colistin monotherapy might be non-inferior when compared to combination therapy used for treating blood stream infections which are caused by carbapenem-resistant gram negative bacilli with the MIC for colistin as $\leq 0.5 \mathrm{mcg} / \mathrm{mL}$ (mortality rate 83 versus 79\%, respectively) [24]. These results have suggested a susceptibility PK/PD breakpoint of $\leq 0.5 \mathrm{mcg} / \mathrm{mL}$ might be able to identify between isolates which are resistant and susceptible to colistin. This suggested breakpoint is less than the present breakpoints (EUCAST) value of $\leq 2 \mathrm{mcg} / \mathrm{mL}$ [11]. However, a prospective cohort clinical study did not find any differences in mortality compared with patients infected by colistin-susceptible and colistin-resistant CRE with $2 \mathrm{mcg} / \mathrm{mL}$ breakpoint [25]. Furthermore, clinical study by Choi, et al. [26] found that the 7-day and 14-day mortality rate in patients who were infected by colistin-susceptible A. baumannii (MIC $<2 \mathrm{mcg} / \mathrm{mL}$ ) with high colistin MICs $(\geq 1 \mathrm{mcg} / \mathrm{mL})$ were statistically significant higher than ones with isolates with lower MICs $(<1 \mathrm{mcg} / \mathrm{mL})$ (38.2\% versus $20.2 \%, p=0.025$ and $35.2 \%$ versus $19.1 \%, p=0.014$, respectively). Therefore, a revision of the current colistin susceptibility breakpoint for Enterobacteriaceae may be needed.

Based on a PK/PD target of $f$ AUC/MIC $\geq 25$, CFR are lower than $90 \%$ for all regimens approved by EMA-, FDA-, Nation et al., Siriraj Hospital in all categories of creatinine clearance. Overall, they are approximately $70-86 \%$. For colistin-resistant isolates, the currently approved regimens achieved CFR target in only $10-50 \%$.

Our simulations were carried out on colistin-susceptible K. pneumoniae and colistin-susceptible E. coli subgroup. For colistin-susceptible K. pneumoniae, this study has shown an attainment rate of $90 \%$ CFR was observed in Siriraj Hospital as well as our study regimens in patients with creatinine clearance $\geq 80 \mathrm{~mL} / \mathrm{min}$. However, CFR targets are achieved for all dosage regimens of patients with creatinine clearance $<80 \mathrm{~mL} / \mathrm{min}$ except the FDA-approved regimens in patients with creatinine clearance 51-79 and 11-29 $\mathrm{mL} / \mathrm{min}$. While, all of dosage regimens achieved CFR target in all renal function groups for colistin-susceptible E. coli infection.

This data supports that colistin is still the mainstay treatment option against carbapenem-resistant K. pneumoniae and carbapenem-resistant E. coli infections provided that: (i) the colistin MIC for the infecting organism is $\leq 0.5 \mathrm{mcg} / \mathrm{mL}$ (ii) high-dose regimens are administered to attain the PK/PD targets. However, high dose of colistin may have adverse effects such as nephrotoxicity and neurotoxicity [27-29]. It is necessary to be careful to balance between risks of toxicity and clinical benefit (iii) for combination of the colistin and other antibiotics, the MICs were markedly lower than the MICs of the antimicrobials in monotherapy and resulting MIC values of colistin $\leq 0.5-1 \mathrm{mcg} / \mathrm{mL}$.

The study has limitations as follows. First, the number of isolates evaluated was not very large and had only two species (K. pneumoniae $(n=96)$ and E.coli $(n=20)$ ). The isolates came from a tertiary university hospital in Bangkok, which might be different from other hospitals or other countries. These are factors which affect the dosing regimen based on the CFR results. Second, we did not simulate about the drug level for patients using hemodialysis. Finally, the molecular characteristics of carbapenemase enzyme has not been performed in this study. 


\section{Conclusions}

Our study is the first to determine effective and compare the colistin dosing regimens approved by the EMA-, FDA-, Nation, et al., Siriraj Hospital and our study against carbapenem-resistant Klebsiella pneumoniae and carbapenem-resistant Escherichia coli. It used differences between the regimens, especially for FDA- provided attainment rates which were lower than other approved regimens. The reduction in the $\mathrm{CrCl}$ improved attainment of \%PTA and \%CFR. Findings indicate the use of alternative dosing approaches allowing for higher daily doses or combination therapy are preferable for patients with $\mathrm{CrCl} \geq 80 \mathrm{~mL} / \mathrm{min}$ and if the MIC of colistin $\geq 0.5 \mathrm{mcg} / \mathrm{mL}$.

Supplementary Materials: The following are available at http://www.mdpi.com/2079-6382/8/3/125/s1, Figure S1: \%PTA of colistin in patients with $\mathrm{CrCl} \mathrm{10-29} \mathrm{(mL/min),} \mathrm{Figure} \mathrm{S2:} \mathrm{\% PTA} \mathrm{of} \mathrm{colistin} \mathrm{in} \mathrm{patients} \mathrm{with}$ $\mathrm{CrCl}<10(\mathrm{~mL} / \mathrm{min})$. Table S1: Colistin dosage regimens for Simulations., Table S2: Our study regimens for Simulations, Table S3: Minimum inhibitory concentrations (MICs) distribution of colistin, Table S4: The recommended dose based on the ability to achieve PTA target at various MICs, Table S5: The recommended dose based on the ability to achieve PTA target at various MICs, Table S6: \%PTA and \%CFR for All isolates, Colistin-susceptible isolates subgroup and Colistin-resistant isolates subgroup. Table S7: \%PTA and \%CFR of our study regimens for All isolates, Colistin-susceptible isolates subgroup and Colistin-resistant isolates subgroup.

Author Contributions: Conceptualization, K.J., P.M. and V.T.; methodology, K.J., P.M., V.T. and J.H.; software, K.J., P.M., K.S., J.H., O.A. and W.S.; validation, K.J., P.M. and K.S.; formal analysis, K.J.; investigation, K.J.; resources, K.J., P.M. and V.T.; data curation, K.J.; writing original draft preparation, K.J.; writing-review and editing, K.J., P.M. and V.T.; visualization, K.J.; supervision, P.M. and V.T.; project administration, K.J., P.M. and V.T.

Funding: This research received no external funding.

Acknowledgments: We would like to thank Stephen Pinder, Faculty of Medicine Ramathibodi Hospital, Mahidol University for his advice on English expression and comments that greatly improved this document.

Conflicts of Interest: The authors declare no conflict of interest.

\section{References}

1. Zarkotou, O.; Pournaras, S.; Tselioti, P.; Dragoumanos, V.; Pitiriga, V.; Ranellou, K.; Prekates, A.; Themeli-Digalaki, K.; Tsakris, A. Predictors of mortality in patients with bloodstream infections caused by KPC-producing Klebsiella pneumoniae and impact of appropriate antimicrobial treatment. Clin. Microbiol. Infect. 2011, 17, 1798-1803. [CrossRef] [PubMed]

2. Munoz-Price, L.S.; Poirel, L.; Bonomo, A.R.; Schwaber, M.J.; Daikos, G.L.; Cormican, M.; Cornaglia, G.; Garau, J.; Gniadkowski, M.; Hayden, M.K.; et al. Clinical epidemiology of the global expansion of Klebsiella pneumoniae carbapenemases. Lancet Infect. Dis. 2013, 13, 785-796. [CrossRef]

3. Nordmann, P.; Naas, T.; Poirel, L. Global Spread of Carbapenemase-producing Enterobacteriaceae. Emerg. Infect. Dis. 2011, 17, 1791-1798. [CrossRef] [PubMed]

4. National Antimicrobial Resistance Surveillance Center, Thailand. Antibiograms. Available online: http: //narst.dmsc.moph.go.th/antibiograms.html. (accessed on 20 July 2017).

5. Kazmierczak, K.M.; Rabine, S.; Hackel, M.; McLaughlin, R.E.; Biedenbach, D.J.; Bouchillon, S.K.; Sahm, D.F.; Bradford, P.A. Multiyear, Multinational Survey of the Incidence and Global Distribution of Metallo- $\beta$-Lactamase-Producing Enterobacteriaceae and Pseudomonas aeruginosa. Antimicrob. Agents Chemother. 2016, 60, 1067-1078. [CrossRef] [PubMed]

6. Hsu, L.Y.; Apisarnthanarak, A.; Khan, E.; Suwantarat, A.; Ghafur, A.; Tambyah, P.A. Carbapenem-Resistant Acinetobacter baumannii and Enterobacteriaceae in South and Southeast Asia. Clin. Microbiol. Rev. 2017, 3, 1-22. [CrossRef] [PubMed]

7. Li, J.; Nation, R.L.; Turnidge, J.D.; Milne, R.W.; Coulthard, K.; Rayner, C.R.; Paterson, D.L. Colistin: The re-emerging antibiotic for multidrug-resistant Gram-negative bacterial infections. Lancet Infect. Dis. 2006, 6, 589-601. [CrossRef]

8. Lim, L.M.; Neang, L.Y.; Anderson, D.; Yang, J.C.; Macander, L.; Jarkowski III, A.; Forrest, A.; Bulitta, J.B.; Tsuji, B.T. Resurgence of Colistin: A Review of Resistance, Toxicity, Pharmacodynamics and Dosing. Pharmacotherapy 2010, 30, 1279-1291. [CrossRef] [PubMed] 
9. Garonzik, S.M.; Li, J.; Thamlikitkul, V.; Paterson, D.L.; Shoham, S.; Jacob, J.; Silveira, F.P.; Forrest, A.; Nation, R.L. Population Pharmacokinetics of Colistin Methanesulfonate and Formed Colistin in Critically Ill Patients from a Multicenter Study Provide Dosing Suggestions for Various Categories of Patients. Antimicrob. Agents Chemother. 2011, 55, 3284-3294. [CrossRef]

10. Clinical and Laboratory Standards Institute. Performance Standards for Antimicrobial Susceptibility Testing. Twenty-Seven Informational Supplement. CLSI Document M100-Swayne; CLSI: Wayne, PA, USA, 2017.

11. European Committee on Antimicrobial Susceptibility Testing (EUCAST). Available online: http:www.eucast. org/clinical_breakpoints/ (accessed on 20 July 2017).

12. Nation, R.L.; Garonzik, S.M.; Thamlikitkul, V.; Giamarellos-Bourboulis, E.J.; Forrest, A.; Paterson, D.L. Dosing Guidance for Intravenous Colistin in Critically Ill Patients. Clin. Infect. Dis. 2017, 64, 565-571. [CrossRef]

13. Tsala, M.; Vourli, S.; Georgiou, P.C.; Pournaras, S.; Tsakris, A.; Daikos, G.L.; Mouton, J.W.; Meletiadis, J. Exploring colistin pharmacodynamics against Klebsiella pneumoniae: A need to revise current susceptibility breakpoints. J. Antimicrob. Chemother. 2018, 73, 953-961. [CrossRef]

14. Cheah, S.E.; Wang, J.; Nguyen, V.T.T.; Turnidge, J.D.; Li, J.; Nation, R.L. New pharmacokinetic/pharmacodynamic studies of systemically administered colistin against Pseudomonas aeruginosa and Acinetobacter baumannii in mouse thigh and lung infection models: Smaller response in lung infection. J. Antimicrob. Chemother. 2015, 70, 3291-3297. [PubMed]

15. Jacobs, M.; Grégoire, N.; Mégarbane, B.; Gobin, P.; Balayn, D.; Marchand, S.; Mimoz, O.; Couet, W. Population Pharmacokinetics of Colistin Methanesulfonate and Colistin in Critically Ill Patients with Acute Renal Failure Requiring Intermittent Hemodialysis. Antimicrob. Agents Chemother. 2016, 60, 1788-1793. [CrossRef] [PubMed]

16. Nation, R.L.; Garonzik, S.M.; Li, J.; Thamlikitkul, V.; Giamarellos-Bourboulis, E.J.; Paterson, D.L.; Turnidge, J.D.; Forrest, A.; Silveira, F.P. Updated US and European Dose Recommendations for Intravenous Colistin: How Do They Perform? Clin. Infect. Dis. 2016, 62, 552-558. [CrossRef] [PubMed]

17. European Medicines Agency Completes Review of Polymyxin-based Medicines: Recommendations Issued for Safe Use in Patients with Serious Infections Resistant to Standard Antibiotics. Available online: https://www.ema.europa.eu/documents/press-release/european-medicines-agency-completesreview-polymyxin-based-medicines_en.pdf (accessed on 2 December 2017).

18. FDA Approved Drug Products. Label and Approval History for Coly-Mycin M, NDA 050108. Available online: https://www.accessdata.fda.gov/scripts/cder/daf/index.cfm?event=overview.process\&ApplNo= 050108 (accessed on 2 December 2017).

19. Eiamphungporn, W.; Yainoy, S.; Jumderm, C.; Tan-Arsuwongkul, R.; Tiengrim, S.; Thamlikitkul, V. Prevalence of the colistin resistance gene mcr-1 in colistin-resistant Escherichia coli and Klebsiella pneumoniae isolated from humans in Thailand. J. Glob. Antimicrob. Resist. 2018, 15, 32-35. [CrossRef] [PubMed]

20. Bradford, P.A.; Kazmierczak, K.M.; Biedenbach, D.J.; Wise, M.G.; Hackel, M.; Sahm, D.F. Correlation of $\beta$-Lactamase Production and Colistin Resistance among Enterobacteriaceae Isolates from a Global Surveillance Program. Antimicrob. Agents Chemother. 2016, 60, 1385-1392. [CrossRef] [PubMed]

21. Maalej, S.; Meziou, M.; Rhimi, F.; Hammami, A. Comparison of disc diffusion, Etest and agar dilution for susceptibility testing of colistin against Enterobacteriaceae. Lett. Appl. Microbiol. 2011, 53, 546-551. [CrossRef] [PubMed]

22. Nation, R.L.; Li, J.; Cars, O.; Couet, W.; Dudley, M.N.; Kaye, K.S.; Mouton, J.W.; Paterson, D.L.; Tam, V.H.; Theuretzbacher, U.; et al. Framework for optimisation of the clinical use of colistin and polymyxin B: The Prato polymyxin consensus. Lancet Infect. Dis. 2015, 15, 225-234. [CrossRef]

23. Nation, R.L.; Velkov, T.; Li, J. Colistin and polymyxin B: Peas in a pod, or chalk and cheese? Clin. Infect. Dis. 2014, 59, 88-94. [CrossRef]

24. Shah, P.G.; Shah, S.R. Treatment and Outcome of Carbapenem-Resistant Gram-Negative Bacilli Blood-Stream Infections in a Tertiary Care Hospital. J. Assoc. Physicians India 2015, 63, 14-18.

25. De Maio Carrilho, C.M.D.; de Oliveira, L.M.; Gaudereto, J.; Perozin, J.S.; Urbano, M.R.; Camargo, C.H.; Grion, C.M.C.; Levin, A.S.S.; Costa, S.F. A prospective study of treatment of carbapenem-resistant Enterobacteriaceae infections and risk factors associated with outcome. BMC Infect. Dis. 2016, 16, 629. [CrossRef] 
26. Choi, I.S.; Lee, Y.J.; Wi, Y.M.; Kwan, B.S.; Jung, K.H.; Hong, W.P.; Kim, J.M. Predictors of mortality in patients with extensively drug-resistant Acinetobacter baumannii pneumonia receiving colistin therapy. Int. J. Antimicrob. Agents 2016, 48, 175-180. [CrossRef] [PubMed]

27. Sorlì, L.; Luque, S.; Grau, S.; Berenguer, N.; Segura, C.; Montero, M.M.; Álvarez-Lerma, F.; Knobel, H.; Benito, N.; Horcajada, J.P. Trough colistin plasma level is an independent risk factor for nephrotoxicity: A prospective observational cohort study. BMC Infect. Dis. 2013, 13, 380. [CrossRef] [PubMed]

28. Horcajada, J.P.; Sorlí, L.; Luque, S.; Benito, N.; Segura, C.; Campillo, N.; Montero, M.; Esteve, E.; Mirelis, B.; Pomar, V.; et al. Validation of a colistin plasma concentration breakpoint as a predictor of nephrotoxicity in patients treated with colistin methanesulfonate. Int. J. Antimicrob. Agents 2016, 48, 725-727. [CrossRef] [PubMed]

29. Falagas, M.E.; Fragoulis, K.N.; Kasiakou, S.K.; Sermaidis, G.J.; Michalopoulos, A. Nephrotoxicity of intravenous colistin: A prospective evaluation. Int. J. Antimicrob. Agents 2005, 26, 504-507. [CrossRef] [PubMed]

(C) 2019 by the authors. Licensee MDPI, Basel, Switzerland. This article is an open access article distributed under the terms and conditions of the Creative Commons Attribution (CC BY) license (http://creativecommons.org/licenses/by/4.0/). 